\title{
Konzepte einer spanischen Bibel- übersetzung des 19. Jahrhunderts in der WLB
}

Die WLB verwahrt im Handschriftenbestand der "Codices biblici in folio" zwei Konzepte einer spanischen Bibelübersetzung, die im Zuge der Abschlussarbeiten am Katalog der Folio-Bibelhandschriften wieder ins Bewusstsein gerückt sind und ein besonderes Augenmerk verdienen. ${ }^{1}$ Es handelt sich dabei um die Übersetzungen der "Versión moderna" des Buchs Genesis (1. Buch Mose) auf 108 Blättern und des Buchs Rut auf sieben Blättern. Interessant daran ist unter anderem ihre Verwahrung unter den Handschriftenbeständen, denn beide Konzepte sind typographisch, also mit der Schreibmaschine geschrieben. Die in der WLB befindlichen Exemplare sind zudem hektographische Kopien.

Die beiden Konzepte mit der Signatur Cod. bibl. $2^{\circ} 87$ (Genesis) und Cod. bibl. $2^{\circ} 88$ (Rut) sind im Jahr 1906 als Bestandteil des geschenkten Nachlasses des Romanisten und evangelischen Theologen Eduard Böhmer (1827-1906) in die WLB gelangt. $^{2}$ Diesem ist das Genesis-Konzept auch gewidmet: „Una nueva traduccion del libro del Génesis. Dr. Edward Boehmer. With compliments of The Translator" (Cod. bibl. $2^{\circ} 87$, fol. 1r). ${ }^{3}$ Der Übersetzer ist der amerikanische Missionar und Lehrer Henry Barrington Pratt (1832-1912), der sich und die Datierung am Ende des Rut-Konzepts auch selbst nennt: "Su hermano y consiervo en J. C., H. B. Pratt. Staunton Va., EE. UU. de America, Set. 29 de 1886" (Cod. bibl. $2^{\circ} 88$, fol. 7v). ${ }^{4}$ Dass es sich bei den Exemplaren um Konzepte handelt, geht aus den handschriftlichen Randbemerkungen und Unterstreichungen hervor, die sich darin finden.

Henry Barrington Pratt wurde am 26. Mai 1832 in der Nähe von Darien im US-Bundesstaat Georgia geboren. Nach dem Studium an der Universität Oglethorpe (Georgia) und am Theologischen Seminar der Universität Princeton (New Jersey) wurde er von der amerikanischen presbyterianischen Kirche ordiniert. Danach war er Missionar in
Bogotá in Kolumbien, sowie an unterschiedlichen Orten in Mexiko, Kuba und in den Vereinigten Staaten unter Menschen hispanoamerikanischer bzw. spanischer Abstammung. In Laredo (Texas) gründete er eine Bibelschule, die Evangelisten und Missionare ausbildete und ihren Schülern dafür nicht nur theologische und pädagogische, sondern auch hauswirtschaftliche Kenntnisse vermitteln wollte. 1899, nachdem die Schule wegen einer Pockenepidemie geschlossen werden musste, wurde er Leiter einer spanischen Gemeinde in Brooklyn (New York). Er starb am 11. Dezember 1912 in Hackensack (New Jersey). ${ }^{5}$

Auf Grund seiner missionarischen und pädagogischen Arbeit begann er ab 1861, zuerst mit dem Spanier Angel H. Mora, eine neue Übersetzung der Bibel ins Spanische zu erstellen. ${ }^{6}$ Das Neue Testament dieser "Versión moderna", eine Revision der Reina-Valera-Übersetzung von 1602 (Übersetzung auf Initiative des Cassiodoro de Reina, um 15201594, mit der Revision des Cipriano de Valera, 1532-1602), erschien als Teil einer Vollbibel 1865. ${ }^{7}$

(1) Codices biblici in folio (Cod. bibl. $2^{\circ}$ 1-90), beschr. von Regina Hausmann, unter Mitarbeit von Carsten Kottmann und Kerstin Losert (Die Handschriften der Württembergischen Landesbibliothek Stuttgart 1,1,1), Wiesbaden 2013. [im Druck]

(2) Zu Böhmer vgl. Friedrich Wilhelm Bautz: Art. Böhmer, Eduard, in: Biographisch-bibliographisches Kirchenlexikon, hg. von Friedrich Wilhelm Bautz, Bd 1, Hamm (Westf.) 1975, Sp. 665.

(3) Eine neue Übersetzung des Buchs Genesis. Dr. Eduard Böhmer, mit Empfehlung des Übersetzers.

(4) ein Bruder und Mitknecht in Jesus Christus, Henry Barrington Pratt, Staunton (Virginia), Vereinigte Staaten von Amerika, 29. September 1886.

(5) Samuel Escobar: Art. Pratt, Henry Barrington, in: Biographical Dictionary of Christian Missions, hg. von Gerald H. Anderson, New York 1998, S. 546f.; R. Douglas Brackenridge / Francisco O. García-Treto: Iglesia Presbiteriana. A History of Presbyterians and Mexican Americans in the Southwest, San Antonio 1974, S. 23-27.

(6) Robert Breaker: A Brief Look at the History of the Spanish Bible, Milton 1999, S. 20. Einen knappen, deutschsprachigen Überblick über die Geschichte der spanischen Bibelübersetzung, allerdings ohne näher auf die Übersetzung von Henry Barrington Pratt einzugehen, bietet Manuel Gutiérrez Marin: Die spanische Bibelübersetzung, in: Die Bibel in der Welt 12 (1969), S. 86-99. Weit umfassender ist die Arbeit von dens., Versiones españolas de la Biblia, Madrid 1958, die aber in deutschen Bibliotheken nicht verfügbar ist.

(7) La Santa Biblia, que contiene et Antiguo y el Nuevo Testamento. Version de Cipriano de Valera, revisada y corregida, New York 1865 (Exemplar der WLB: B span 1865 02); vgl. Bernd Bauske: i(Sl\{c)\}h]chibboleth! Eine Untersuchung zur Wiedergabe der Schibboleth-Episode (Richter 12,6) in der spanischen Bibeltradition, Stuttgart 1997, S. 36-38 
1884 begann Pratt, nunmehr ohne Mora, auch das Alte Testament zu übersetzen. Diese Übersetzungsarbeiten wurden zumindest teilweise von der American Bible Society finanziert, die die Übersetzung später auch in mehreren (Teil-)Ausgaben verlegte. $^{8}$

Über die näheren Umstände der Konzepte sind wir über einen Brief Pratts an Eduard Böhmer vom 9. März 1886 informiert, der sich heute im Nachlass Eduard Böhmers in der Universitätsbibliothek Heidelberg befindet. ${ }^{9}$ Darin entschuldigt sich Pratt dafür, dass seine Antwort (Pratt und Böhmer standen schon länger in einem Briefwechsel) so lange habe auf sich warten lassen und dass er ein "bad correspondent" sei; aber die Arbeit an der spanischen Bibelübersetzung halte inn mit einem tyrannischen Griff fest, doch habe er weder den Willen noch die Kraft, sich davon zu befreien. ${ }^{10}$ Die amerikanische Bibelgesellschaft sei gerade dabei, den Druck der Genesis-Übersetzung vorzubereiten, die auf der hektographischen Kopie beruht, die Pratt Böhmer

(8) Brief an Eduard Böhmer vom 11. Juli 1885 (Heidelberg, UB, Heid Hs. 2379); Brackenridge/García-Treto, Iglesia (wie Anm. 5), S. 23.

(9) Heidelberg, UB, Heid. Hs. 2379.

(10) "[...] this work of Spanish Bible translation holds me with a tyrannical grasp, from which I have neither will nor power to disengage myself".

(11) "You will be gratified to learn that the Am. Bible Soc. have now almost all in type an edition of Genesis based on the hektograph copy sent you [...]".

(12) El Génesis, nuevamente traducido del original Hebreo, y que reproduce, lo mejor que se puede, el propio estilo de Moisés, New York 1886 (vgl. Historical catalogue of the printed editions of the Holy Scripture in the library of the British and Foreign Bible Society, Bd 2, bearb. von Thomas H. Darlow und Horace F. Moule, London 1911, Nr. 8595); das Ex. der WLB: B span 1886 02. - Die Übersetzung des Buchs Rut findet sich in der Vollbibel-Ausgabe: La Santa Biblia. Constando del Antiguo y el Nuevo Testamento. Traducida de las lenguas originales, y cotejado diligentemente con muchas y diversas traducciones, versión moderna, New York 1893 (vgl. Historical catalogue of the printed editions ..., Nr. 8613); das Ex. der WLB mit der Signatur B span 189301 ebenfalls mit Widmung: Dr. Ed. Boehmer with compliments of the translator H. B. Pratt. Matamoros Mex [Mexico] Dec 81893.

(13) Für Dr. Eduard Böhmer. Mit Empfehlungen der Achtung und Wert schätzung, von seinem Freund Henry Barrington Pratt, Übersetzer. Staunton (Virginia) 26. Januar 1887.

(14) Zu ihm vgl. A. Gordon Kinder: Casiodoro de Reina: Spanish Reformer of the Sixteenth Century, London 1975; Jorge A. González: Cassiodoro de Reina. Traductor de la Biblia en español, Mexico 1969.

(15) Eduard Böhmer: Spanish reformers of two centuries from 1520 Their lives and writings, according to Benjamin B. Wiffen's plan and with the use of his materials, Bd. 2, Straßburg/London 1883, S. 174.

(16) Natürlich, falls das Alte Testament noch nie aus dem hebräischen Original durch christliche Hände übersetzt worden ist, dann heißt das: Je früher es gemacht wird, wenn es denn gut gemacht wird, desto besser".

(17) Brief an Eduard Böhmer vom 11. Juli 1885 (Heidelberg, UB, Heid. Hs. 2379). Auf seine eigene Übersetzungsleistung aus dem Hebräischen wird in seiner Genesis-Übersetzung, die er 1886 herausgibt, ausdrücklich verwiesen: nuevamente traducido del original hebreo (vgl. Anm. 12). - Details zu seinem Übersetzungsprogramm auch in Henry Barrington Pratt: Estudios criticos y aclaratorios fundados en la version moderna, New York 1903. Das Exemplar der WLB (Signatur: Theol.oct. 14116) ist ebenfalls Eduard Böhmer gewidmet.

(18) Vgl. Anm. 12 geschickt hatte und sich eben nun als Cod. bibl. $2^{\circ} 87$ in der WLB befindet. ${ }^{11}$ Von diesem Druck, den Pratt hier anspricht, befindet sich ein Exemplar in der Bibelsammlung der WLB;12 auch dieses ist Eduard Böhmer gewidmet: "To DR. EDWARD BOEHMER. With sentiments of regard \& esteem, from his friend H. B. Pratt, Translator. Staunton Va. [Virginia, USA] Jan 26th 1887“.13

In seinem Brief erwähnt Pratt weiter, dass es einige Stimmen gäbe, die eine moderne spanische Bibelübersetzung nicht für notwendig erachten. Grund dafür sei die weitverbreitete Überzeugung, dass der Initiator der ersten reformatorischen spanischen Bibelübersetzung von 1569, Cassiodoro de Reina, ${ }^{14}$ das Alte Testament direkt aus dem Hebräischen übersetzt habe - was Pratt anzweifelt. Diesen Zweifel habe auch schon Böhmer gehegt, wie dieser im zweiten Band seines Buchs „Spanish reformers" erwähnt. ${ }^{15}$ Angesichts einer anstehenden Revision der de Reina-Übersetzung gibt es für Pratt daher keinen Grund, eine Bibelübersetzung zu revidieren, die ihrerseits selbst nur eine Übersetzung einer Übersetzung ist. Aus diesem Defizit zieht Pratt nun seine Schlussfolgerung: „Of course, if the Old Testament has never been translated [...] out of the Original Hebrew, by Christian hands, then the sooner it can be done, if well done, the better". ${ }^{16}$ Dies ist die Begründung und Legitimation für seine eigene Bibelübersetzung aus dem hebräischen Original, die er 1884 begonnen hatte. ${ }^{17}$

Diese neue Übersetzung, deren Konzept in den oben genannten Handschriftenbänden vorliegt, wurde von Böhmer wohl gründlich durchgeschaut. Zumindest unterscheidet sich der Druck der Genesis-Ausgabe an etlichen Stellen vom Konzept, wie es in Cod. bibl. $2^{\circ} 87$ vorliegt. Das gleiche Ergebnis ergibt ein Vergleich der Rut-Übersetzung aus Cod. bibl. $2^{\circ} 88$ mit der Fassung in der Vollbibel-Ausgabe von Pratt aus dem Jahr 1893. ${ }^{18}$ Ein Vergleich beider Versionen könnte weitere Hinweise zur Übersetzungsleistung Pratts und ihrer Bedeutung für die Geschichte der spanischen Bibelübersetzung im 19. Jahrhundert liefern.

Carsten Kottmann 